\title{
Laser-based Time Transfer through Free-space Links
}

\author{
Ivan Prochazka, \\ Josef Blazej, Tereza Flekova, Jan Kodet \\ Czech Technical University in Prague, Brehova 7, 11519 Prague 1, Czech Republic
}

The two-way time transfer is an effective way to synchronize two independent time scales with high precision and accuracy independently on the variations of the interconnecting channel [1]. We are reporting on a new approach to an optical two-way free space time transfer which is based on signals of individual photons. This approach enables to reach extreme timing stabilities and minimal systematic errors using existing electro-optic technologies. In our previous work we have demonstrated electronic circuits for two-way time transfer via a single coaxial cable with picosecond accuracy and precision [2]. We have designed and tested the optical analogy of the two-way time transfer using a common optical channel. Photon counting approach to the signal detection is providing several key advantages: the reduction of most systematic errors found in commonly used multi-photon detection systems and the capability to operate with ultimately low signals. The repetitive optical signals of an average intensity as low as $1 \times 10^{-4}$ photon per pulse may be detected and time tagged with sub-picosecond precision and stability [3]. The principle is illustrated in figure 1.

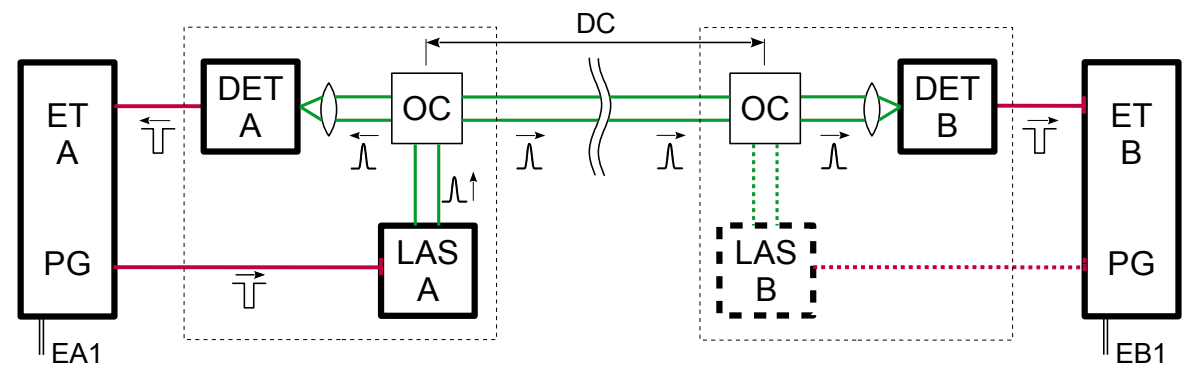

Fig. 1 Block scheme of optical two-way time transfer. Red lines stand for electrical signal lines, green lines stand for optical signal paths. The first measurement phase when module $A$ is generating optical signals is displayed.

Two event timers $E T A$ and $E T B$ are equipped with identical optical modules. The event timers represent the time scales $A$ and $B$. Each optical module consists of an optical signal combiner $O C$, laser optical source $L A S$ and a photon counting detector $D E T$. These modules are interconnected by an optical channel: free space in our case. The measurement is carried out in two phases. First the laser $L A S A$ is generating an ultrashort optical pulse. It is split by a signal combiner $O C A$ and is propagating in both directions toward $D E T A$ and $D E T B$. They are detected by these detectors and time tagged in the respective time scales $A$ and $B$. The corresponding epochs are recorded and stored. In the second phase the laser $L A S B$ is generating ultrashort uniform pulse, which is split and propagating toward both detectors DET $A$ and DET B. The corresponding epochs are recorded. Two pairs of epochs can be combined producing the time difference $D S$ between the two time scales along with the delay of the optical channel $D C$ interconnecting the two optical modules. Reference points are defined symmetrically inside each optical signal combiner with picosecond accuracy.

The performance and capabilities of described scheme was demonstrated in a number of ground experiments. The limiting precision was better than $400 \mathrm{fs}$, the long term stability was better than 1 ps over days of operation. The accuracy of optical two way time transfer better than 3 ps was achieved. Analogical two way time transfer was completed via optical fiber which replaced the free space signal propagation. The timing performance achieved was similar to a free space one.

Laser time transfer based on photon counting approach is used also for ground to space time scales comparison. Such a scheme is an extension of the existing satellite laser ranging. Its great advantage is that it completely compensates all the influence of Earth atmosphere and troposphere delays down to sub-picosecond level. Several space missions based on photon counting laser time transfer were successfully completed last years. Recently European Space Agency [4] and China are preparing two space mission with laser time transfer ground to space comparison of atomic and optical clocks on ground and onboard space stations.

\section{References}

[1] J. Levine, "A review of time and frequency transfer methods," Metrologia 45, S162-S174 (2008).

[2] P. Pánek, J. Kodet, and I. Procházka, "Accuracy of two-way time transfer via a single coaxial cable," Metrologia 50, 60-65 (2013).

[3] I. Procházka, J. Kodet, and J. Blažej, "Note: Solid state photon counters with sub-picosecond timing stability," Review of Scientific Instruments 84, 046107 (2013).

[4] M. P. Heß, L. Stringhetti, B. Hummelsberger, K. Hausner, R. Stalford, et al., "The ACES mission: system development and test status," Acta Astronautica 69, 929 (2011) 